\title{
« Hi I'm a Liberal, hi I'm a PC », web social et publicité comparative en politique
}

Benoit Cordelier et Pauline Breduillieard

\section{(2) OpenEdition}

12 Journals

Édition électronique

URL : http://journals.openedition.org/communicationorganisation/3774

DOI : 10.4000/communicationorganisation.3774

ISSN : 1775-3546

Éditeur

Presses universitaires de Bordeaux

Édition imprimée

Date de publication : 1 juin 2012

Pagination : 131-145

ISBN : 978-2-86781-821-9

ISSN : 1168-5549

\section{Référence électronique}

Benoit Cordelier et Pauline Breduillieard, « «Hi I'm a Liberal, hi I'm a PC », web social et publicité comparative en politique », Communication et organisation [En ligne], 41 | 2012, mis en ligne le 01 juin 2015, consulté le 01 mai 2019. URL : http://journals.openedition.org/

communicationorganisation/3774 ; DOI : 10.4000/communicationorganisation.3774 


\title{
«Hi I'm a Liberal, hi l'm a PC", web social et publicité comparative en politique
}

\author{
Benoit Cordelier', Pauline Breduillieard²
}

Gerstlé (1993) définit la communication politique comme : "l'ensemble des techniques et procédés dont disposent les acteurs politiques [...] pour séduire, gérer et circonvenir l'opinion ». Ainsi, le marketing politique aussi assimilé à de la communication politique est souvent considéré et qualifié de propagande moderne (Albouy, 2000). L’idée politique est devenue un message médiatique et pour cela les spécialistes de la communication politique se sont inspirés des techniques marketing et commerciale de la publicité. Pour Cialdini (1990, 2004), l'apparence est une des grandes forces de persuasion en psychologie sociale, ainsi les hommes politiques font façonner leur image par des publicitaires et des relationnistes comme ils façonneraient une image de marque. De ce fait, la communication politique est principalement devenue la capacité d'un politicien à gérer son image (Gerstlé, 1993). Ces emprunts à la communication commerciale ont changé le visage du débat politique et l'image du politicien.

En 2007, lors de la campagne électorale au Canada, les Jeunes libéraux ont tourné une campagne publicitaire qui récupérait certains codes d'une série de publicités d'Apple. Par sa mise en perspective, nous tenterons de voir alors comment la communication politique évolue, en s'inspirant de la communication commerciale, afin d'atteindre un public désabusé et dont les modalités d'engagement se renouvellent.

Dans une première partie, nous présenterons les origines de la publicité politique et les emprunts croisés avec la publicité commerciale en abordant plus particulièrement le cas des publicités comparatives et des negative spots. Ensuite, dans une deuxième partie, nous introduirons la campagne de 2007 des Jeunes libéraux canadiens qui illustre clairement cette volonté du politique à renouveler leurs modalités de communication en se servant des médias

1 Chaire de relations publiques et communication marketing, Université du Québec à Montréal, Canada ; cordelier.benoit@uqam.ca

2 Chaire de relations publiques et communication marketing, Université du Québec à Montréal, Canada ; breduillieard.pauline@uqam.ca 
sociaux et de la publicité en tant que référence populaire. Enfin, dans une troisième partie, nous approfondirons l'analyse du mécanisme démonstratif jouant sur la connivence qu'elle met en place.

\section{Publicité commerciale et publicité politique : parcours croisés}

\section{Une brève histoire}

La communication marketing et la communication politique, toutes deux des communications d'influence, partagent des racines communes depuis les recherches d'Harold Lasswell, de Paul Lazarsfeld et d'Elihu Katz. Les travaux de ces deux derniers en particulier, à travers les livres The People's Choice (1944) et Personal Influence (1955) révèlent des mécanismes d'influence qui fonctionnent aussi bien dans les phénomènes de consommation que d'élaboration des préférences politiques. Dans la foulée, 1952 est considérée comme la date de début du marketing politique avec la campagne pour l'élection présidentielle des Etats-Unis à laquelle Eisenhower était candidat. Celui-ci avait fait appel à une agence de publicité et à des relationnistes pour façonner son image auprès des citoyens-électeurs (Albouy, 2000). Les procédés utilisés en marketing des biens et services ont ainsi été intégrés à la politique : études du marché politique, sondages d'opinion, création d'un slogan. Les agences de publicité ont été de plus en plus sollicitées et ont favorisé l'influence de la communication commerciale vers la communication politique à travers notamment une simplification, voire une sloganisation des messages politiques. Selon, Albouy (2000, p. 7) cette « simplification, transposant le principe commercial de la proposition unique de vente (Unique Selling Proposition), s'accompagne d'un recours systématique au spectaculaire et à l'émotionnel ».

Peu après, des publicités plus agressives, dénigrant l'adversaire, ont commencé à être développées : les negative spots. Les premiers sont apparus en 1956 avec la fameuse campagne How's that again, General ? des démocrates qui attaquaient le président sortant Eisenhower. Ainsi, les conseillers publicitaires et politiques, en s'inspirant également de la publicité comparative, ont décidé de montrer le candidat adverse de manière négative en utilisant des extraits de discours où il faisait des promesses qu'il n'avait pas tenues (Albouy, 2000). Nous aborderons plus en détail ces deux types de publicité ultérieurement.

Suite à ces débuts, le marketing politique a dépassé les frontières américaines pour apparaître sur la scène politique européenne et dans d'autres pays. Elle est par exemple arrivée en France en 1965 avec le candidat aux élections présidentielles Jean Lecanuet et l'association de François Mitterrand et Jacques Séguéla fait encore école avec le slogan. D'ailleurs, si le slogan de la campagne de 1981, La force tranquille, est emprunté à Léon Blum et Jean Jaurès, celui de 1986, Génération Mitterrand, ferait un clin d'œil à Génération Pepsi, d'une célèbre boisson pétillante. 
Après s'être développé par le recours aux agences publicitaires et l'utilisation des médias de masse, le marketing politique s'adapte aujourd'hui en intégrant aux campagnes politiques les médias électroniques (Internet, réseaux sociaux numériques) qui avaient été plus rapidement préemptés par la publicité commerciale (Maarek, 2007). Cela a été particulièrement remarqué en 2007 avec la campagne électorale américaine de Barack Obama.

\section{Emprunts croisés}

Selon Gerstlé (1989), les principales techniques publicitaires qui ont été reprises dans le discours politique sont la réduction et la clarification des contenus et la «sloganisation » du message ce qui permet d'avoir de courtes phrases et des formules adaptées à la diffusion dans les médias. De ce fait, la publicité politique s'inspire du marketing commercial comme le mentionne Albouy (2000, p22) : "le marketing politique pourrait servir à désigner un ensemble de théories, de méthodes, de techniques et de pratiques sociales, inspirées du marketing commercial, qui sont toutes destinées à persuader les citoyens d'apporter leur soutien à un homme, à un groupe ou à un projet politique ». Les dimensions argumentatives et démonstratives de la communication politique en sortent donc transformées.

Lorsque l'on observe certaines publicités commerciales et politiques, nous pouvons facilement avancer que l'une et l'autre s'inspirent mutuellement, on peut parler d'emprunts croisés entre la politique et la publicité commerciale (Adam, 1981). Pour développer leurs activités, les publicitaires ont envahi la sphère politique en devenant les forgerons de l'image des politiciens. Durant les années 50-70, il y a eu de la part des publicitaires une exploitation du monde politique qui s'est faite par une dépolitisation de la communication politique (Souchier, 1992). Les arguments politiques ont quasiment disparu pour laisser la place au politicien et à son image. Il était même fréquent de le mettre en scène comme cela serait fait pour un produit de consommation. A maintes reprises une publicité commerciale a été adaptée à une publicité politique. Maarek (2007) nous expose un exemple éloquent avec la campagne qui avait été réalisée pour le candidat républicain au Sénat, Malcolm Wallop, dans l'état du Wyoming en 1976. Dans son message, le candidat était représenté en train de faire une chevauchée sur un cheval blanc. Ce spot publicitaire était certes en lien avec l'image que le sénateur voulait donner et une manière de toucher les citoyens de cet État reconnu comme étant l'État du rodéo, mais il s'agissait aussi d'une publicité rappelant celles faites pour la marque Marlboro.

Toutefois, ces emprunts ne se sont pas faits que dans un seul sens, car dans les années 80-90 la publicité commerciale s'est, à son, tour inspirée du discours politique comme nous le démontre Souchier (1992) en présentant une publicité de Volkswagen qui reprend une version adaptée du drapeau de l'Union soviétique. Cette publicité a été diffusée en 1991-92 au moment même 
où l'Union soviétique disparaissait. L'affiche use de symboles politiques pour promouvoir la Golf de Volkswagen. La faucille et le marteau sont inversés et remplacés par un vilebrequin et une clé à molette. L'étoile jaune laisse place au mot « Niet » et nous retrouvons en bas de l'affiche le logo de la marque de voiture, le tout apposé sur un chiffon rouge qui rappelle certes le chiffon du mécanicien mais surtout la couleur du drapeau de l'ex-URSS. Plus près de nous, en 2011, un fabricant israélien de produits de nettoyage de voiture s'est servi dans ses publicités des visages de Nicolas Sarkozy et de Barak Obama pour essuyer des pare-brises en écho à l'incident diplomatique où les deux hommes ont traité Benjamin Netanyahu de « menteur ». Cette même année, Benetton a lancé une nouvelle campagne de shockvertising dans laquelle des chefs politiques de même sexe mais aux relations conflictuelles s'embrassaient goulûment sur la bouche.

Ces croisements d'univers sont intéressants le partage d'un contexte socio-culturel instaure ainsi une certaine connivence entre le consommateur qui est également un citoyen et le produit faisant l'objet de la publicité. C'est le même mécanisme qui est à l'œuvre dans la publicité politique. Par la reconnaissance de l'univers quotidien du citoyen, l'homme politique cherche ainsi à montrer son inscription dans le siècle et sa proximité avec le reste de la population.

\section{Des publicités comparatives aux negative spots}

Il existe différents types de publicité commerciale. L'un d'eux est nommé publicité comparative. Nous retrouvons beaucoup ce genre de publicité en Amérique du Nord, par exemple aux États-Unis, elle représentait dans les années quatre-vingt un tiers de tous les messages publicitaires (Stewart et Furse, 1986). Cette proportion est restée la même de nos jours. Née en 1930 aux États-Unis, la publicité comparative est, comme le mentionne Miskolczi-Bodnár (2004), un dispositif de promotion qui compare les produits d'un annonceur avec ceux d'un autre ou plusieurs autres concurrents. Le rôle de ce genre de message est de mettre en avant les avantages du produit de l'annonceur par rapport à son concurrent et ainsi de souligner les faiblesses du compétiteur.

Il existe deux sortes de publicités comparatives (Wilkie et Farris, 1975 ; Jackson, Brown et Harmon, 1979). Les premières sont celles qui comparent au moins deux marques du même produit en les citant. Elles peuvent le faire de façon explicite ou implicite. Dans ce cas, nous pourrons parler, de la part de l'annonceur, de dénigrement du concurrent puisqu'il met en lumière les défauts de ce dernier. Alors que les deuxièmes comparent les marques sur les attributs spécifiques du produit en s'appuyant sur des superlatifs tels que le numéro un du marché, etc. Ici, les concurrents ne sont pas cités directement.

Bien que très présente en Amérique du Nord depuis longtemps, la publicité comparative est apparue en Europe, plus tardivement, dans les années 80 
et est depuis 2000 très réglementée. Toutefois, elle n'y est pas bien perçue, notamment en France, en Allemagne, en Italie ou encore au Luxembourg (Dianoux et Herrman, 2000).

De plus, même si nous avons vu que dans certains pays la publicité comparative semble avoir quelques difficultés à faire ses marques, il n'en reste pas moins que dans ceux où elle est très présente, elle semble être appréciée des consommateurs. Malgré cela, certains annonceurs jugent opportun d'ajouter à ce type de publicité un peu d'humour pour désamorcer le dénigrement du concurrent. Ainsi, l'annonceur conserve une image positive aux yeux du public. Un exemple connu et récent, est la campagne de publicités d'Apple (2006-2009) intitulée Mac vs PC. Dans cette campagne que nous aborderons plus longuement dans la partie suivante, Apple dénigre avec humour Microsoft en « ringardisant » le PC face à un Mac jeune et souriant.

Bien que la publicité commerciale fût la source première d'inspiration de la publicité politique, il n'en existe pas moins des différences entre les deux types de discours (Adam, Bonhomme, 2007). Le fait que le message politique puisse être comparatif, et critique, voire dénigrant, est mieux accepté en politique où l'approche plus conflictuelle semble davantage adaptée au style de communication et le sauver la face (Goffman, 1955) moins attendu par le public. La nature du débat dans l'espace public (Habermas, 1962, 1992), même en tenant compte des critiques qui lui sont faites (Fraser, 2001) se prêterait davantage à ce genre de traitement. C'est pourquoi la publicité politique peut être techniquement inspirée de la publicité commerciale (Adam, 1981), tout en adoptant des exécutions qui y seraient davantage polémiques. En communication politique, nous retrouvons une variante des publicités comparatives sous le terme de negative spots ou publicité négative. Comme nous l'avons mentionné précédemment, ces derniers sont apparus aux EtatsUnis dans les années 50. Gerstlé (1992) définit ce style publicitaire ainsi : "Les spots négatifs, ensuite, visent à discréditer l'identité ou les propositions de l'adversaire en procédant par allusion, comparaison ou attaque directe. "Ce genre de publicité est monnaie courante aux États-Unis puisque comme la publicité commerciale comparative, environ un tiers des campagnes politiques utilisent cette technique comme le mentionne Sabato (1981). Une des principales raisons qui fait que ces publicités fonctionnent aussi bien est que, selon Owen (1991), les votants aiment et désirent connaître les différences qui existent entre les candidats et, par l'intermédiaire des negative spots, ils obtiennent cette information.

Il existe ici plusieurs déclinaisons de cette publicité politique négative qui s'inspirent des publicités comparatives commerciales. Miller et Gronbeck (1994) en identifient trois sortes (nous gardons ci-après les expressions anglaises) : les implicative - qui fonctionnent grâce à des insinuations et n'attaquent pas directement l'adversaire - les comparative - qui mettent de l'avant, juxtaposent les promesses ou les prises de positions des candidats - et, 
enfin, les assault - qui ont pour rôle d'attaquer directement l'adversaire, ses actions et ses associés. Ainsi, les publicités négatives permettent d'exposer clairement les différences qui existent entre le candidat et son adversaire, mais aussi d'attirer les votes des électeurs comme le mentionne Mark (2009, p. 4) : "Going negative on the opponent is the best way to draw clear differences and run on the issues the challenger favors. ». Dans son livre, Mark (2009) souligne l'importance d'utiliser l'humour dans les campagnes négatives. Car selon lui, l'intégration de l'humour ou encore de l'irrévérence dans les negative spots aura un impact plus important sur l'électeur puisque ce dernier est souvent exposé aux publicités négatives. Cela permettra aussi de faire passer le message de façon moins brutale. Pour justifier ce point, il cite les propos du spécialiste média des Démocrates américains, Jim Margolis (Mark, 2009, p. 5) : «Humor ... can be a very effective way to make a point, and connect on an emotional level, which ... has more impact than sort of a tic-list of failures"

La publicité négative, ou negative spots, se pose donc comme une variante de la publicité comparative commerciale. Elle en reprend les techniques de persuasion mercatique. Toutefois, il faut noter que la communication politique jouit de plus de latitude que celle commerciale comme l'explique Albouy (2000, p. 176) : "le message publicitaire politique dispose d'une plus grande liberté d'expression que son homologue commercial, dans la mesure où il peut être comparatif, négatif et même impunément mensonger, le comparatisme et le négativisme étant ici justifiés par la nature même des enjeux. ».

\section{La campagne 2007 des Jeunes libéraux canadiens}

À partir d'une analyse de contenu comparative de la campagne des Jeunes libéraux du Canada, une branche du Parti Libéral canadien, inspirée d'une campagne d'Apple, nous verrons quels sont les discours et les figures rhétoriques qui sont empruntés et adaptés de la publicité commerciale d'origine, et en quoi cela amincit la frontière entre la communication politique et la communication commerciale. À travers cet exemple, nous essaierons de montrer comment la publicité politique cherche, sans perdre de son mordant, à rejoindre un public qui lui échappe.

\section{Hi, I'm a Liberal}

La campagne Hi, I'm a Liberaß ${ }^{3}$, composée de trois spots, a été réalisée par les Jeunes libéraux du Canada et diffusée sur l'internet lors de la campagne électorale de 2007. Ces trois messages publicitaires suivent la même séquence. Les spots s'ouvrent sur les deux protagonistes qui se présentent chacun à leur tour : une jeune femme et un jeune homme représentant, respectivement, les Jeunes libéraux du Canada et le Parti conservateur du Canada. La

3 http://hi.im.a.liberal.ca/video.aspx?ad=branding consulté le 11 janvier 2012. 
jeune femme est dynamique, bien habillée tout en restant détendue (jeans, petite veste noire et t-shirt rouge) et sûre de ce qu'elle veut. De l'autre côté, nous avons un jeune homme qui semble plus confus et dont la tenue vestimentaire, un costume-cravate mal agencé, reflète son attitude. S'en suit un jeu de questions-réponses dans lequel le jeune homme pour se justifier use d'arguments positifs pour tenter de valoriser ses expédients, tandis que la jeune femme, elle, énonce quelques pistes de solutions. À chacune des idées proposées par cette dernière, le jeune homme répond par un refus franc et réaffirme sa position. Le message termine par le logo des Jeunes libéraux du Canada accompagné de son adresse internet.

Également, dans ces messages nous retrouvons une approche relativement complexe du message qui devrait se baser sur le principe de simplification à travers une proposition unique de vente, principe clé de la publicité commerciale. Les Jeunes libéraux dénoncent la simplification des messages du Parti Conservateur (PC) tout en laissant entendre qu'ils appréhendent mieux la complexité des enjeux. Leur proposition unique de vente devient alors la suivante : le PC vous leurre en vous proposant des expédients, alors que nous, libéraux, apportons des pistes de solution. L'originalité n'est décidément pas dans le message mais, comme nous le verrons, dans sa forme.

En effet, il est à noter que cette campagne rappelle très clairement celle d'Apple $^{4}$ : Mac vs $P C$, car elle s'en approprie l'esthétique et suit une séquence très proche. Dans le cas d'Apple, les messages débutent avec un jeune homme décontracté et branché qui se présente en disant : "Hello, I’m a Mac » et un autre qui représente un homme un peu plus âgé en costume-cravate avec des lunettes, qui ressemble au stéréotype du comptable ou du bureaucrate, et dit à son tour " and I'm a $P C$ ». Dans chacune des publicités de Mac, $\mathrm{PC}$ essaye de se valoriser par rapport à Mac mais au final il n'y arrive jamais et se rend ridicule par ses comportements. Un autre point commun que nous pouvons signaler c'est l'utilisation dans l'ensemble des spots d'une ritournelle qui rappelle fortement celle que l'on peut trouver dans la campagne Mac vs PC.

Le premier spot, intitulé Branding, démarre avec la jeune fille qui salue et dit « Hi, I'm a Liberal » puis le jeune homme répond " $H$ i, I'm a $P C$, well I was a PC then I was in Canadian Alliance and now I'm just a Conservative». $\mathrm{PC}$ explique que ces changements de nom sont une technique de marketing: «Marketing, my friend, simply marketing» (0'10"). Ces manœuvres superficielles sont là pour installer la confusion dans l'esprit des électeurs et permettre aux Conservateurs de garder le pouvoir. De l'autre côté, la jeune libérale trouve cela anormal et pense au contraire qu'il faut essayer de convaincre les électeurs avec des idées. Par ce message, les Jeunes libéraux veulent informer les jeunes que les Conservateurs les trompent en changeant de nom et que c'est plutôt

4 http://adweek.blogs.com/adfreak/get-a-mac-the-complete-campaign.html consulté le 11 janvier 2012. 
avec une présentation et une défense des idées que l'on peut vraiment aider les citoyens.

Le deuxième spot Global Warming ${ }^{5}$ débute avec la même phrase introductive: «Hi, I'm a Liberal» «Hi, I'm a $P C$ ». Il aborde la thématique du réchauffement climatique. PC, vêtu d'une combinaison de pêche, explique cyniquement qu'il s'agit de l'unique solution envisageable pour combattre la montée des eaux. À l'inverse, la jeune libérale énonce plusieurs solutions et actions réalistes à mettre en place. À nouveau dans ce spot, les Jeunes libéraux nous montrent un Parti conservateur ridicule (par sa tenue, symbolisant leurs expédients, et son style d'argumentation) qui se moque des problèmes de société et qui n'a pas de réelles idées politiques. Tandis que les libéraux, eux, ont une pensée plus articulée et essayeront de trouver des solutions. Cette publicité semble être la plus négative à l'égard du Parti conservateur dans cette campagne.

Enfin, le dernier spot, Cross Dressing ${ }^{6}$, présente cette fois le conservateur vêtu de la même façon que la jeune femme. En se travestissant (au propre comme au figuré), $\mathrm{PC}$ veut faire croire à l'électorat qu'il a des idées progressistes, et ainsi récupérer des intentions de vote. La jeune libérale, en partie flattée, lui fait remarquer que c'est un style qui lui va bien : «You know, it's an okay look for you » vu qu'il prétend embrasser les idées libérales. Face à ce commentaire, $\mathrm{PC}$, énervé, explique que dès qu'il obtiendra la majorité aux élections, il laissera tomber ce masque et réaffirmera ses vraies valeurs, celles des Conservateurs. La jeune libérale énonce clairement que ce n'est pas le genre de transformation qu'elle veut voir. Par cette conclusion, indirectement elle lance un appel aux électeurs à voter Libéral. Ainsi avec ce spot, les Jeunes libéraux veulent montrer aux citoyens que les Conservateurs les ont déjà trompés en faisant semblant de croire en des valeurs importantes pour les Canadiens, et qu'ils sont prêts à recommencer pour accéder au pouvoir.

\section{Comédie et negative spot}

La production des spots des Jeunes libéraux reprend les caractéristiques de ceux d'Apple, de la forme au style. Toutefois, elle est pour le moins maladroite et souffre d'une comparaison directe avec les spots d'Apple. La qualité de l'image, la lumière froide, le cadrage plus rapproché, la musique au registre légèrement strident (bien que fortement inspirée de celle d'Apple) donnent une forte sensation d'amateurisme. Au-delà de ces faiblesses, l'objectif de ces publicités est bien évidemment d'intéresser les jeunes canadiens à voter pour le Parti libéral en allant les chercher dans leur univers. En effet, les jeunes Canadiens tendent à se sentir moins enclins à voter que leurs aînés (Turcotte, 2007). Ce n'est pas tant qu'ils ne s'intéressent pas à l'action politique mais plutôt que leur forme d'engagement est différente (MacKinnon, Pitre et Watling, 2007). Les partis politiques ne sont plus aujourd'hui représentatifs

5 http://hi.im.a.liberal.ca/video.aspx?ad=carbonbudget consulté le 11 janvier 2012.

6 http://hi.im.a.liberal.ca/video.aspx?ad=crossdressing, consulté le 13 avril 2011. 
de la manière dont les jeunes souhaitent s'engager. Ceux-ci l'ont compris et cherchent à les rejoindre, que ce soit à travers leur organisation principale ou leurs branches "jeunesse " en s'emparant de leurs canaux et style de communication. Nous nous rappellerons, par exemple, du lipdub suranné des jeunes UMP en France en 2009. L'exercice n'est pas facile mais reflète bien les efforts pour aller chercher les jeunes et leur rendre plus attrayant un univers politique conventionnel qu'ils dédaignent. Investir l'internet fait partie des recommandations qui sont faites aux structures politiques (MacKinnon, Pitre et Watling, 2007 ; Young et Cross, 2007), même si elles ne disent pas grand-chose sur les spécificités du style de communication à adopter.

Le style de publicité choisi par les Jeunes libéraux correspond donc au grand classique américain des negative spots. Il faut néanmoins retenir que les Canadiens sont sensibles au sauver la face de manière générale aussi bien qu'en publicité (Breduillieard, 2010). L'arène politique traditionnelle se prête pourtant moins à ce type de préoccupation. Les affrontements peuvent y être violents et les dénigrements fréquents, d'autant plus en période électorale. Par ailleurs, les negative spots sont une tactique risquée car la publicité négative n'est pas toujours bien reçue par les électeurs (Pinkleton, 1997) et, comme ils sont malgré cela devenus monnaie courante, leur effet tend à diminuer et doit être amélioré par une hybridation du style avec d'autres éléments comme l'humour (Mark, 2009). La campagne de publicité comparative d'Apple Mac vs $P C$ correspond à ces critères. Car si c'est une publicité comparative critique de PC, elle a eu l'intelligence d'adopter un style humoristique et de faire endosser le caractère critique et désagréable au personnage qui incarne PC. La tactique est fine et difficile à contrer. La force de l'approche d'Apple est donc de laisser croire qu'ils sont suffisamment à l'aise pour ne pas s'encombrer de critiques qui ne leur apportent rien.

Les spots de la campagne des Jeunes libéraux essayent de récupérer la même stratégie et les mêmes mécanismes. La justification comique est établie dès la première vidéo, Branding, où ils jouent sur l'homonymie des initiales communément employées pour Personal Computer et PC qui était, jusqu'en 20037, le sigle du Progressive Conservative Party of Canada. Ils mettent donc en scène deux personnages. L'un représente les conservateurs sous les traits d'un jeune homme un peu déphasé, excité, opportuniste, cynique et se contentant d'expédients aux problèmes qui se posent à la société canadienne. L'autre, représentant les libéraux, est une jeune femme un peu plus posée, positive, empathique et proposant même des solutions au jeune conservateur (qui évidemment les balaye du revers de la main). Non seulement elle n'a pas besoin de critiquer l'autre personnage qui se ridiculise par son attitude même,

7 Le parti conservateur (Conservative Party) a été fondé en 1867 et est devenu le premier parti de gouvernement ou d'opposition. En 1942, il change de nom pour Progressive Conservative Party of Canada. En 2003, il est dissout et ses membres rejoignent le Conservative Party of Canada formé par les membres de la Canadian Alliance. Le nom de Progressive Conservative (PC) reste néanmoins utilisé dans la plupart des provinces canadiennes. 
mais elle est également suffisamment généreuse pour être force de proposition à son égard. Elle signifie ainsi que ses idées sont plus importantes que son éventuelle réussite électorale. Le jeu des acteurs insiste sur le paralangage de chacun des personnages pour renforcer les effets et les idées que nous venons d'exposer. Chacun des personnages reprend l'essentiel de la gestuelle mercatique que détaille Albouy (1994, p. 232). Le jeune conservateur adopte plus particulièrement une attitude désordonnée, gesticulante qui montre sa mauvaise maitrise de soi et par rebond souligne son inconsistance politique. À l'inverse, la jeune libérale est ouverte, à l'écoute et explique avec autant de précision que peut le permettre un spot d'environ 60 secondes. L'effet comique ainsi obtenu désamorce l'agressivité de la critique et permet même de justifier l'invraisemblance du personnage du jeune homme conservateur qui, avec ses cheveux en bataille et sa tenue débraillée, ne correspond pas au stéréotype des jeunes conservateurs au complet-cravate bien repassé et les cheveux soigneusement coiffés comme le casque immuable de Stephen Harper ${ }^{8}$.

Un des points intéressant réside dans le discours suffisamment élaboré de la jeune libérale pour donner l'impression qu'elle maîtrise les sujets qu'elle aborde et être ainsi crédible. Cela est rendu possible par la longueur des spots et est notamment visible dans celui intitulé Global Warming sur le développement durable. Elle contraste positivement par rapport à son interlocuteur qui se préoccupe plus d'effets de communication ("Marketing, my friend, simply marketing » dit-il dans le spot Branding pour expliquer les incessants changements de nom de son parti). La campagne essentiellement émotionnelle cherche toutefois un équilibre dialectique par l'emploi de mots-marqueurs qui montrent bien le clivage politique. Le fait de convoquer les accords de Kyoto, qui, déjà en 2006, dérangeaient pour le moins les conservateurs canadiens ${ }^{9}$, en est un bon exemple.

\section{Syllogismes, implicite et interprétation}

Les publicités d'Apple peuvent être interprétées comme une sorte de démonstration où sont mises en scène des caractéristiques qui différencient le PC et les Mac. Celle-ci peut alors, dans une certaine mesure, être analysée comme la spectacularisation d'une logique formelle par un processus narratif. Chez Aristote, le syllogisme est la forme qui permet la démonstration. Dans les Premiers analytiques, il en avance cette définition : "Le syllogisme est une énonciation dans laquelle certaines propositions étant posées, on en conclut nécessairement quelque autre proposition différente de celles-là, par cela seul que celles-là sont posées. » Un syllogisme s'articule autour de trois éléments : une prémisse majeure, une prémisse mineure et une conclusion. Nous retrouvons

8 Stephen Harper est le Premier ministre du Canada élu en 2006. Il est aussi le chef du Parti conservateur du Canada.

9http://www.ledevoir.com/politique/canada/129295/harper-a-deja-qualifie-kyoto-de-complot-socialiste, consulté le 13 avril 2011. 
bien les bases de ce modèle dans la publicité d'Apple. L'analyse peut essentiellement être menée avec l'inclusion d'une prémisse négative (tab. 1).

\begin{tabular}{|l|c|c|c|}
\hline \multirow{3}{*}{ Prémisse majeure } & \multicolumn{2}{|c|}{ germes } \\
\cline { 2 - 4 } & $\begin{array}{c}\text { ges ordinateurs de bureau } \\
\text { (PC sousWindows Micro- } \\
\text { soft })\end{array}$ & sont & à problèmes. \\
\cline { 2 - 4 } & petit & or & moyen \\
\hline Prémisse mineure & Les ordinateurs Mac & ne sont pas & à problèmes. \\
\cline { 2 - 4 } & petit & donc & grand \\
\cline { 2 - 4 } Conclusion & Les ordinateurs Mac & ne sont pas & $\begin{array}{c}\text { des ordinateurs de } \\
\text { bureau (comme les } \\
\text { autres PC sous Win- } \\
\text { dows Microsoft). }\end{array}$ \\
\cline { 2 - 4 } & & & \\
\hline
\end{tabular}

Tableau. 1 : Les syllogismes d'Apple

Les publicités de Mac mettent l'accent sur le caractère exceptionnel de leur produit en jouant de la comparaison qui met le syllogisme à l'épreuve de l'implicite. En effet, puisque les Mac sont en fait des ordinateurs de bureau, cette publicité laisse aux spectateurs le soin de compléter plusieurs éléments du message. Tout d'abord, ils sont juges de la supériorité du produit Mac qui n'est presque jamais ouvertement affirmée ; ils sont amenés à conclure au caractère exceptionnel des ordinateurs d'Apple. Deuxièmement, l'association $\mathrm{PC} / \mathrm{Microsoft}$ est laissée à l'appréciation du spectateur. Personne n'est dupe et nous indiquons bien l'interprétation évidente en signalant l'implicite entre parenthèses dans le tableau illustrant le syllogisme. Mais, de cette façon, la confrontation n'est pas directe et permet de laisser une porte ouverte au sauver la face. Troisièmement, il est intéressant de constater que la publicité Mac n'aboutit pas sur une invitation directe à l'achat : les spots se ferment sobrement sur une image d'écran d'ordinateur avec le nom «Mac » en dessous. Il n'y a donc pas d'incitation directe à l'achat. Le spectateur est d'une certaine manière laissé libre d'exercer son esprit critique. Après tout, la publicité d'Apple ne fait que reprendre des défauts, connus de tous les usagers, des ordinateurs fonctionnant sous Windows. Linterprétation, ou décodage (Hall, 1980), est suffisamment guidée dans les saynètes pour qu'Apple ne prenne pas grand risque. Au contraire, l'entreprise parvient à préserver une image conviviale grâce à la bonhomie et à l'empathie dont fait preuve le personnage qui l'incarne dans le spot.

Cette ouverture laissée à l'interprétation du récepteur est certes une tactique pour atténuer la violence des propos mais elle a également pour conséquence 
un affaiblissement du cheminement logique du syllogisme. En effet, alors que les Mac sont des ordinateurs de bureau, nous arrivons quasiment à une conclusion inverse. Ce paradoxe, autrement dit ce paralogisme, est résolu par l'interprétation du spectateur qui a bien compris que les PC sont en fait des ordinateurs avec le système d'exploitation Windows de Microsoft. Théoriquement, au moins la prémisse majeure d'un syllogisme doit être universelle. Dans l'exemple qui nous occupe, ce n'est finalement pas le cas. Dans la prémisse majeure, nous avons un cas particulier (les ordinateurs sous Windows), tout comme dans la prémisse mineure (les ordinateurs Mac). I1 n'est donc pas possible de tirer de conclusion formelle en suivant la narration de ces publicités. Si la démonstration est finalement efficace, c'est qu'elle permet la construction de deux idées parallèles qui sont reliées par un implicite que décode le spectateur grâce à sa maîtrise d'éléments contextuels externes. La publicité ne fonctionne par conséquent pas ici grâce à des éléments de logique formelle mais, plutôt, par une approche inductive à partir de laquelle le spectateur infère les conclusions vers lesquelles l'annonceur veut le guider.

Terminons maintenant sur la campagne des Jeunes libéraux. Ils tentent par l'intermédiaire de l'humour d'intéresser les jeunes à la politique et de les faire adhérer aux idées du Parti libéral canadien. En se réappropriant une publicité à succès, ils ont voulu jouer sur un transfert des qualités reconnues à cette campagne et aux produits Apple. Mais, au-delà de ce clin d'œil, ils jouent encore plus que la publicité d'origine sur un semblant de raisonnement logique. Le syllogisme tient encore moins que dans la publicité d'Apple puisqu'ici sont clairement exposés deux cas particuliers : celui du Parti conservateur et celui du Parti libéral ; les deux sont ouvertement mis en parallèle. Cette publicité est somme toute plus agressive que son inspiration commerciale car la confrontation rhétorique n'est plus masquée. Le seul mécanisme qui adoucit l'attaque est l'humour calqué sur celui de la publicité Mac. La dimension de sauver la face, bien que présente grâce à cela, n'est pas prépondérante et ancre ces messages plutôt du côté des negative spots. L'équilibre ainsi obtenu entre agressivité et bienveillance feinte permet aux Jeunes libéraux de se valoriser un peu plus aux yeux du public car, des deux partis, ils se présentent évidemment comme les seuls ayant de vraies pistes de solution à proposer. Ils jouent donc sur deux tableaux : celui idéologique dénonçant les positions de leurs opposants et celui de la complicité générée par le partage de codes communs à travers la reconnaissance plus qu'assumée de la publicité d'Apple.

\section{Conclusion}

La communication politique moderne, inspirée de techniques aujourd'hui attribuées au marketing, date d'une soixantaine d'années. Elle répond au besoin sans cesse croissant des politiciens de rejoindre un électorat qui leur échappe. 
C'est tout particulièrement le cas des jeunes électeurs qui rejettent les modes traditionnels de communication politique (Milan, 2005; MacKinnon, Pitre et Watling, 2007 ; Turcotte, 2005, 2007). L'utilisation des médias est un élément crucial dans la réflexion pour atteindre les publics, et l'Internet est considéré, aujourd'hui, comme un des moyens de communication les plus importants pour toucher les jeunes (MacKinnon, Pitre et Watling, 2007). Ce constat fait, il n'est pas pour autant évident de trouver le style de communication qui permet d'intéresser les jeunes électeurs.

L'effort fait par les Jeunes libéraux canadiens est à ce titre intéressant : ils s'inspirent d'une publicité comparative très appréciée par leur cible (dans le sens marketing du terme) tout en s'inscrivant dans une tradition relativement récente des negative spots. Cela leur permet de travailler sur deux registres. Tout d'abord par l'humour et la référence à un univers ancré dans la société de consommation, ils développent une dimension ludique qui favorise une ouverture communicationnelle au sens de Bougnoux (1998). Le défi pour les Jeunes libéraux étant ici d'attirer l'intérêt des jeunes tout en apparaissant comme étant suffisamment sérieux et crédibles pour inscrire leur message dans l'univers politique. Deuxièmement, dans cette optique, ils parviennent à garder une légitimité en respectant dans une certaine mesure les codes de la communication politique à travers la mise en scène d'un modèle argumentatif logique. Sur ce deuxième point, nous avons néanmoins remarqué que la forme de la logique classique n'est pas respectée en faveur d'une approche plus inductive. Le travail d'interprétation ainsi favorisé incite le spectateur à rentrer dans une dynamique de complicité active et facilite des transferts de représentations en cascade. Ici, le rire favorise des empathies et l'adhésion à des personnages, puis éventuellement à des idées. De cette manière, le registre de la connivence et de l'empathie cherche à rejoindre celui de la démonstration logique.

Les évolutions de la communication politique n'ont cessé d'inquiéter les penseurs de notre société contemporaine. Pour certains, le détachement des individus de la politique en lien avec l'aliénation de la société de masse est une manifestation d'une certaine forme de désintérêt à l'égard de leurs semblables (Arendt, 1961). Pour d'autres, l'évolution des formes du débat dans l'espace public (Habermas, 1962) amène une instrumentalisation du potentiel acclamatif des masses. Dans nos sociétés démocratiques, la lutte pour le pouvoir passe pourtant par un intéressement et une implication minimale de l'électorat. Les partis politiques rentrent à ces fins dans une course à l'électorat très fortement basée sur leurs actions de communication. Celles-ci s'inspirent des techniques de la communication marketing pour séduire et contrer le désenchantement des électeurs. Mais il ne faudrait pas pour autant oublier les relations ambivalentes qu'entretiennent déjà les consommateurs avec la publicité (Cordelier et Toffoli, 2009). 
D'autres pistes, d'autres manières d'envisager la politique sont donc à considérer (Milan, 2005 ; MacKinnon, Pitre et Watling, 2007 ; Turcotte, 2005, 2007) afin d'échapper au consumérisme politique.

\section{BIBLIOGRAPHIE}

ADAM J-M., «Votez Mir Rose, achetez Giscard : Analyses pragmatiques », Pratiques, 1981, $\mathrm{n}^{0} 30$.

ADAM J-M. et BONHOMME M., L'argumentation publicitaire. Rhétorique de l'éloge et de la passion, Paris, Armand Colin, 2007.

ALBOUY S., Marketing et communication politique, Paris, L'Harmattan, Logiques sociales, 2000 [1994].

ARENDT H., La condition de l'homme moderne, Paris, Calmann-Levy, 1961

BOUGNOUX D., Introduction aux sciences de la communication, Paris, La Découverte, 1998.

BREDUILLIEARD P., Efficacité de la publicité bi-référentielle au Mexique, Sarrebruck, Éditions Universitaires Européennes, 2010.

CIALDINI R., Influence et manipulation, Paris, First documents, 2004 [1990].

CORDELIER B. et TOFFOLI R., Perception de la publicité - Une comparaison France Québec, Rapport de recherche. Chaire de relations publiques et communication marketing, Université du Québec à Montréal, 2009.

DIANOUX C. et HERRMANN J-L., " La Publicité Comparative en Europe : Bilan et Perspectives ", In Convegno "Le tendenze del marketing in Europa", 24 novembre, Venise, Italie, 2000.

FRASER N., « Repenser la sphère publique : une contribution à la critique de la démocratie telle qu'elle existe réellement », Hermès, vol. 31, 2001.

GERSTLÉ J., "La communication et la dualité public/privé ", Revue française de science politique, $37^{\mathrm{e}}$ année, $\mathrm{n}^{\circ} 5$. pp. 659-674, 1987.

GERSTLÉ J., La publicité politique - Quelques enseignements de l'expérience américaine. Hermès 4, Ed. CNRS, 1989.

GERSTLÉ, J., La communication politique, Que sais-je ? Paris, PUF, 1993.

GOFFMAN E., "On facework », Psychiatry, 18, 213-231. - repris par JOO YUP K., SANG HOON N., "The Concept and Dynamics of Face: Implications for Organizational Behavior in Asia », Organization Science, Vol. 9, no 4, pp. 522-534, 1955.

HABERMAS J., L'espace public : archéologie de la publicité comme dimension constitutive de la société bourgeoise, Paris, Payot, 1962, réed.1993.

HABERMAS J., De l'éthique de la discussion, Paris, Cerf, 1992.

HALL S., " Coding and encoding in the television discourse », In S. Hall et al. (Eds.), Culture, media, and language, pp. 197-208, London, Hutchinson, 1980.

JACKSON D. W. Jr., BROWN S. W. et HARMON R. R., « Comparative Magazine Advertisements ", Journal of Advertising Research, 19 (December), pp. 21-26, 1979.

KATZ E. et LAZARSFELD P. F., Personal Influence, New York, Free Press, 1955. 
LAZARSFELD P. F., BERELSON B. et GAUDET H., The People's Choice. New York, Duell, Sloan and Pearce, 1944.

MAAREK P.J., Communication et marketing de l'homme politique, Paris, Litec (3e Ed.), 2007.

MACKINNON M.P., PITRE S. et WATLING J., " Lost in Translation: (Mis) Understanding Youth Engagement », Rapport de recherche, Canadian Policy Research Networks, 2007.

MARK D., Going dirty : the art of negative campaigning, New York, Rowman \& Littlefield, 3e ed., 2009.

MILLER A.H. et GRONBECK B.E. (eds), Presidential campaign and American self images, Boulder, CO, Westview, 1994.

MISKOLCZI-BODNÁR P., "Definition of comparative advertising ", European Integration Studies, Vol. 3. (1), pp. 25-44, 2004.

OWEN D., Media messages in American presidential elections, New York, Greenwood, 1991.

SABATO L.J., The Rise of Political Consultants: New Ways of Winning Elections, New York, Basic Books, 1981.

SOUCHIER E., "La publicité comme détournement du politique ", In : Communication et langages, N93, 3ème trimestre, pp. 36-51, 1992.

STEWART D. W. et. FURSE D. H., Effective TV Advertising: A Study of 1000 Commercials, Lexington, MA, Lexington Books, 1986.

TURCOTTE A., " "What Do You Mean I Can't Have A Say?” Young Canadians and Their Government », Rapport de recherche, Canadian Policy Research Networks, 2007.

WILKIE W. L. et FARRIS P., "Comparison Advertising : Problems and Potential », Journal of Marketing, 39 (October), pp.7-15, 1975.

YOUNG L. et CROSS W., "A Group Apart: Young Party Members in Canada », Rapport de recherche, Canadian Policy Research Networks, 2007.

Résumé : depuis les années 50 , la politique emprunte les techniques de communication utilisées en marketing pour promouvoir les politiciens et leurs partis. En prenant pour exemple la campagne de 2007 des Jeunes libéraux du Canada, nous montrerons comment la publicité politique essaye d'attirer un public a priori désintéressé, les jeunes, en s’inspirant d'une publicité commerciale.

Mots-clés : communication politique, internet, marketing, negative spots, publicité comparative.

Abstract : Since the 50's, political communication draws on marketing techniques to promote politicians and their parties. We use here as an example the Young Liberals of Canada campaign from 2007 to show how, mimicking a popular commercial, political advertising tries to draw the attention of a seemingly disinterested public of young people

Keywords : comparative advertising, internet, marketing, negative spots, political communication, rhetoric. 
